\title{
How surface and fomite infection affect contagion dynamics: a study with self-propelled particles
}

\author{
Sayantari Ghosh $^{1, \mathrm{a}}{ }_{\mathbb{D}}$, Arijit Chakraborty ${ }^{1}$, and Saumik Bhattacharya ${ }^{2}$ \\ 1 Department of Physics, National Institute of Technology, Durgapur, India \\ 2 Department of E\&ECE, Indian Institute of Technology, Kharagpur, India
}

Received 29 October 2021 / Accepted 18 December 2021 / Published online 12 January 2022

(C) The Author(s), under exclusive licence to EDP Sciences, Springer-Verlag GmbH Germany, part of Springer Nature 2022

\begin{abstract}
Self-propelled particles have been a tool of choice for many studies for understanding spatial interaction of people and propagation of infectious diseases. Other than the direct contagion process through face-to-face contacts with an infected agent, in some diseases, like COVID-19, the disease can spread by indirect ways, through contaminated object surfaces and puff-clouds created by the infected individual. However, this dual spreading process and the impact of these indirect infections in the entire dynamics are not properly explored. In this work, we consider epidemic spreading in an artificial society, with realistic parameters and movements of people, along with the possibilities of indirect exposure through contaminated surfaces and puff-clouds. This particular simulation based infectious disease dynamics is associated with the movements of some self-propelled free agents executing random motion which is investigated in conjunction with the rules of a realistic contagion process. With mathematical formulation and extensive computational studies, we have accommodated the indirect infection possibilities into the dynamics by incorporating an infectious 'tail' with the infected individuals. Analytical expressions of survival distance and infection probability of individuals have been explicitly calculated and reported. Results of precise and comparative simulation study have revealed the seriousness of indirect infections in connection with several dynamical parameters. Using this framework, interpretation of multiple waves in local as well as global scenarios have been established for COVID-19 infection statistics. Furthermore, the importance of indirect infections are also pointed out through data fitting, showing that ignoring this component might cause a misinterpretation of the dynamical parameters, like, imposed restrictions.
\end{abstract}

\section{Introduction}

The virus SARS-COV2 and the disease caused by it, COVID-19, has widely affected almost all the countries across the globe. At present nearly 240 million people have been already exposed to the virus worldwide. Though several vaccines are invented to increase the immunity against the virus, it is evident that social distancing and travel restrictions are very effective as intervention strategies for the infection spread. Several theories have already been considered to explore the probable ways of transmission of the virus through social contacts. To emphasize the importance of contact-based spreading further, World Health Organization (WHO) has already exposed the reports [1] related to the transmission of coronavirus through surface contamination or via a close contact with an infected person. The airborne aspect [2-6] of coronavirus has also been studied through extensive experiments to understand the overall risk. It is recently revealed that the droplets from an sick person

\footnotetext{
${ }^{a}$ e-mail: sayantari.ghosh@phy.nitdgp.ac.in (corresponding author)
}

could stay on air from few minutes to several hours, and can contribute significantly in the infection spreading of COVID-19 $[2,3]$. The other modes of transmission like fomites (objects or materials which are likely to carry infection) and surface infection can also contribute directly in the spreading [7-12]. These two different modes of infection transmissions are shown in Fig. 1 using simple schematics.

In the context of this pandemic, tremendous efforts were observed from researchers on data based approaches that relied mostly on the available data in government repositories as well as on social media platforms [1316]. Data-driven fractal-based approaches that dealt with the time series of infection spread have provided deeper insights as predictive models [17-19]. However, the most popular approach of modeling an epidemic is based on early works of Kermack and McKendrick [20] using ordinary differential equations (ODE) by partitioning the population into SIR (susceptible-infectedrecovered) compartments. The transition between these subpopulations are analyzed to understand the overall dynamics of the infection spread. Clever extensions of the basic classical idea to stochastic as well as spatial 


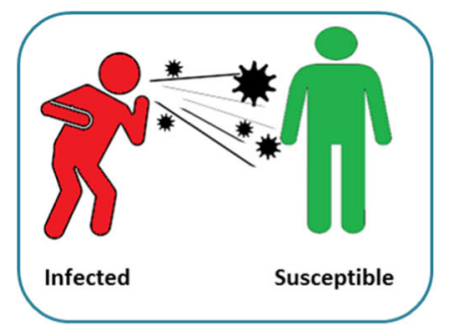

Direct Contagion

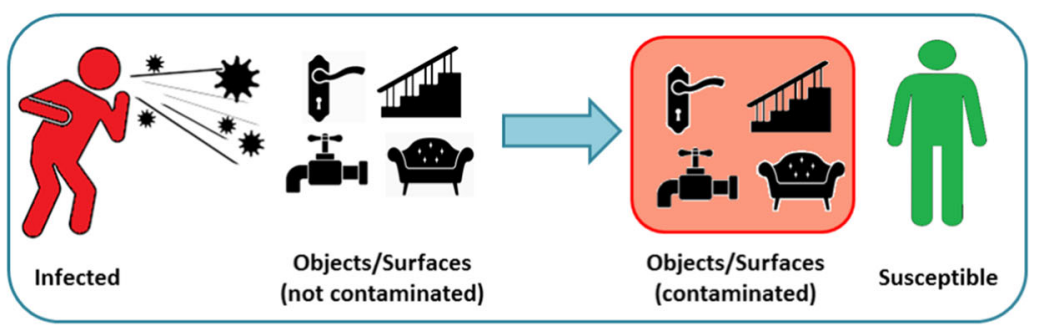

Indirect Contagion Process

Fig. 1 Schematic representation of the proposed idea where indirect contagion process also contributes in disease spreading in certain infectious diseases, like COVID-19

aspects have been very effective for predictions for the current as well as the past pandemics [21-25].

In a different approach, the spreading process of the epidemic dynamics has been also analyzed through some random, self-propelled particles, moving and interacting in a synthetic society [23,26-28]. As people often move and interact without following any deterministic or probabilistic rules, thus, the interactions and movements of a group be well approximated using Brownian motion-like Dynamics (BMD) [23]. However, in an epidemiological dynamics, unlike standard Brownian motion, an interaction between two self-propelled particles not only changes their movements but also causes the transmission of the disease following the infection model, with SIR-like contagion rules as backbone. The implementation of a standard infection model, like SIR, divides the particles into three kind of health status (susceptible, infected and recovered). A close contact of any infected particle with a susceptible one results to a transformation of the health status of the susceptible particle to infected or exposed. However, such transition from susceptible to infected state can also be considered as a probabilistic transitions depending on different factors like immunity, nature of the disease, distance etc. [29]. Recently, such BMD-based technique has gained popularity in analyzing COVID-19 dynamics as it spreads mainly through contacts. Geometric Brownian motion has proven to be very effective to model the real data of daily infection cases. The spread of the infection through geometric Brownian motion has been studied in the context of COVID-19 contagion in Italy [30]. In [23], the propagation of the infection is discussed through close contact of Brownian particles with three kind of different health status (based on SIR model). An expression of microscopic contagion rate over the population density is also considered using mean-free path analysis of 2D randomized motion. An investigation on contagion dynamics in a system of self-propelled agents is discussed in [31]. Using basic SIR model, the outbreak thresholds were analyzed by the authors among active particles in different contagious conditions.

Interestingly, though the transmission of the infection through BMD is fairly analyzed in different papers, the effects of indirect contamination through fomites and surface infection have not been analyzed in any existing study. However, with spatial aspect involved in the dynamical process, BMD can work nicely as the baseline model to implement surface contamination effects. indirect infections, like droplets and fomites, play a significant role to increase the infection rate as people often become ignorant about these risks as there might be no sick person present on spot. Though these factors play significant roles in infection transmission $[32,33]$, often they are ignored in mathematical models that have explored to study transmission dynamics. In this work, we have focused on the indirect mode of infection transmission along with the contact-based transmission process, and have extensively analyzed the effects of such indirect transmissions on overall infection dynamics. As WHO report [1] has already contagion the significance of the infection spread through indirect contacts, it is immensely important to analyze the role of indirect infections, other than direct contact between two agents to have a more realistic models of COVID-19 infection spread.

In this paper, we aim to highlight the importance of considering the indirect infection along with the personto-person based direct infection. This helps us to analyze the infection spreading in a more realistic scenario. The major contributions of the work are as follows.

- We propose a BMD-based epidemic dynamics considering both direct and indirect contacts. To the best of our knowledge, this is the first work that has considered indirect infection spreading with BMD framework for a more realistic analysis.

- We establish a mathematical foundation for the proposed study and implement the dynamics on a comprehensive computational platform for comprehensive and thorough analysis.

- We perform extensive computational simulations and show that the consideration of the indirect infection yields significantly different outcomes, even with the same transmission parameters.

- To demonstrate the effect of indirect infection clearly, we also fit the real data of COVID-19 infection with the proposed framework. We found that the consideration of indirect infection can significantly change our understanding of the transmission dynamics. Thus, ignoring this factor might cause an unrealistic estimation of model parameters. 
The rest of the paper is organized as follows. The methodology has been described in Sect. 2 whereas the results consisting of the comparative analyses, parametric studies, data understanding and interpretation are reported in Sect. 3. Finally, we conclude our work in Sect. 4 by discussing the key observations and future scopes of the proposed model.

\section{Methodology}

In this section, we discuss the mathematical design and the simulation framework of the proposed model. The parameter settings that are used for analyzing the framework are also discussed in detail.

\subsection{Model formulation}

To consider the possibility of movements and role of indirect infection in this dynamics, we take this model contagion process in a $2 \mathrm{D}$ synthetic population where individuals are free to move and interact with other individuals in the society. We consider a square arena of side length $L \in \mathbb{R}$ with reflective boundary condition containing $N \in \mathbb{Z}^{+}$number of active individuals executing active BMD. At a particular time instant, the $j$ th particles have a direction of movement indicated by vector $\hat{\mathbf{r}}_{j}=\left(\cos \left(\theta_{j}\right), \sin \left(\theta_{j}\right)\right)$. However, this direction can change in case of collision with other particles, or with the wall. Spontaneous change in the direction of movement can also be allowed considering a random angle after a time $\zeta$, i.e., $\theta_{j}=\theta_{j}^{t}$ for $(n-1) \zeta<t<n \zeta$ can be assigned a new value $\theta_{j}=\tilde{\theta}_{j}^{t}$, at $t=n \zeta\left(n \in \mathbb{Z}^{+}\right)$, causing a random reorientation of each self-propelled particle.

Now to incorporate the infection spread in this interactive synthetic society, we use the rules similar to that of SIR model [20]. In this ODE based model, individuals belong to compartments called, susceptible, infected, recovered; $S, I$ and $R$ denote the fraction people who are in susceptible, infected and recovered susceptible health status in the population at time $t$. It is assumed that the population maintains a fixed size without any birth or death rates. The overall characteristics of the epidemic is featured in two simple transition rules:

$$
S \stackrel{\beta}{\longrightarrow} I, \quad I \stackrel{\gamma}{\longrightarrow} R
$$

Here, $\beta$ is transmission or infection rate of the disease and, $\gamma$ is recovery rate for the epidemic. Our infection spread follows a similar process; however, to consider a range of infection spread, each self-propelled particle is assumed to have a sphere of influence whose projection on the $2 \mathrm{D}$ space has a radius $r_{n} \in \mathbb{R}, n \in N$ and a direct infection spread happens if for particles $p_{i}$ and $p_{j},\left\|\mathbf{c}_{\mathbf{i}}-\mathbf{c}_{\mathbf{j}}\right\|_{2} \leq\left(r_{i}+r_{j}\right)$ where $\mathbf{c}_{\mathbf{i}}$ and $\mathbf{c}_{\mathbf{j}}$ are the centers of particles $p_{i}$ and $p_{j}$ respectively and $i \neq j$. Here, $\|\cdot\|_{2}$ indicates $l_{2}$ norm of a vector. In other words, if $s_{p_{i}}(t)$ indicates the projection of sphere of influence of

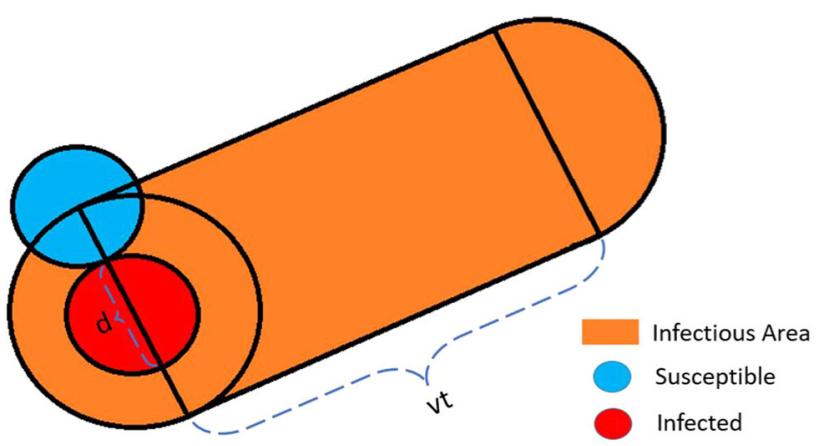

Fig. 2 Schematic representation of total infectious area of an infected individual considering only direct infection spread

an infected particle $p_{i}$, then a susceptible particle $p_{j}$ will be infected iff $s_{p_{j}}(t) \cap s_{p_{i}}(t) \neq \phi$, where $s_{p_{j}}(t)$ is the projection of the sphere of influence of the susceptible particle $p_{j}$, and $\phi$ is the empty set.

To understand the dynamics of the indirect infection, let us assume that the fomites spread by an infected individual can be active for $t^{\prime}$ time interval from generation, and if any susceptible person comes into the contact of the fomites in that duration, it becomes infected even without having any direct contact with an infected person. Moreover, we assume that a self-propelled infected individual is constantly creating fomites and causing surface infection until it is recovered. Now, a susceptible agent $p_{j}$ will be infected by an contagious particle $p_{i}$ considering both direct and indirect infections, iff:

$$
s_{p_{j}}(t) \cap \tilde{s}_{p_{i}} \neq \phi \text {, where } \tilde{s}_{p_{i}}=s_{p_{i}}(t)+\int_{t-t^{\prime}}^{t} s_{p_{i}}(\omega) \mathrm{d} \omega .
$$

Here, the first term of $\tilde{s}_{p_{i}}$ covers the direct infection and the second term covers the indirect infection due to fomites and surface-contagions. It is evident that as $t^{\prime}$ increases, the indirect infection contributes significantly in the infection spreading.

Now, if $n_{i}^{t}$ is the number of infected individuals in the arena at time $t$, any interaction with the susceptible individuals might result into a new infected case, causing $n_{i}^{(t+1)}=n_{i}^{t}+p_{c} n_{s}^{t}$, where $p_{c}$ is the probability of infection and $n_{s}^{t}$ is the number of susceptible people. The quantity $p_{c}$ might also be considered as the collision probability of an infected and a susceptible individual, which can be extrapolated to the mean distance traveled before such an encounter. This distance, $L_{d}$, is referred as survival distance for the rest of the text.

Without loss of generality, let us assume that all the particles have same sphere of influence with diameter $d$. Considering particles traveling with an average speed of $v$, we now calculate the $L_{d}$ based on assumptions similar to [23]. Contact area of a single infected individual is $2 d v t+\pi d^{2}$. The schematic illustration in Fig. 2 shows 


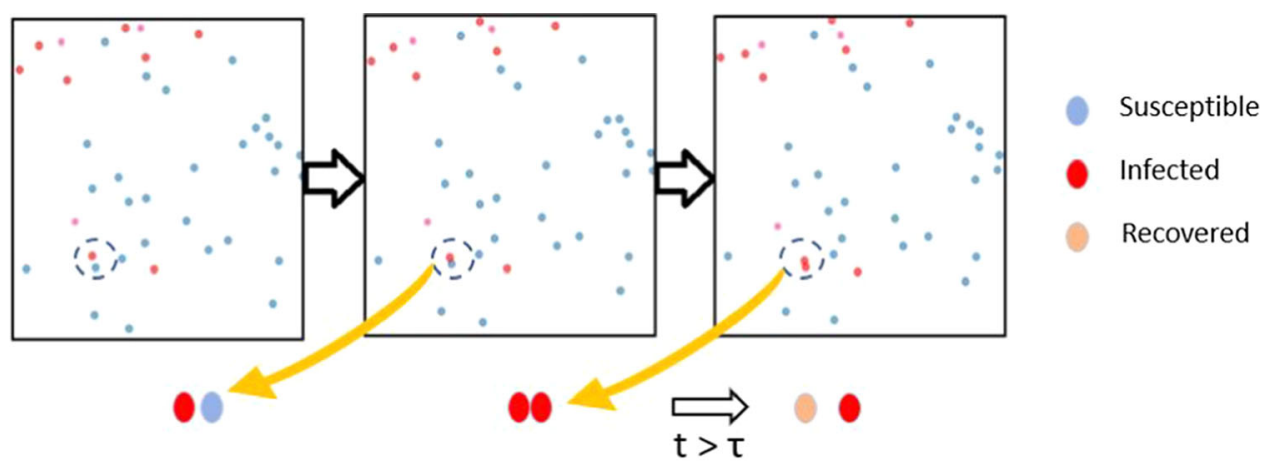

(a)

(b)

Fig. 3 A series of snapshots from the simulation considering self propelled particles with only direct infections. a In the simulation arena size is $1.5 \times 1.5$. $N$ active, self propelled, without infectious tail particle present. b A close con-

the movement of a particle of diameter $d$ for a time duration $t$, with a velocity $v$, and the area of contact it covers. Thus, the survival distance by considering only the collisions of the particle is,

$$
L_{d}=\frac{v t}{\left(2 d v t+\pi d^{2}\right) n_{v}},
$$

where $d$ is the diameter of sphere of influence of each infected individual and $n_{v}=\frac{n_{s}^{t}}{L^{2}}$, is the number density of susceptible people.

Now, if a tail of infection of last $t^{\prime}$ time steps is still effective, then the survival distance changes as,

$$
L_{d}^{\prime}=\frac{v t}{\left(2 d v\left(t+t^{\prime}\right)+\pi d^{2}\right) n_{v}} .
$$

Thus, the infectious area of the arena as well as infection probability, $p_{c}=A_{i n} / A$ (where $A=L^{2}$, and $A_{\text {in }}=n_{i}^{t}\left(2 d v\left(t+t^{\prime}\right)+\pi d^{2}\right)$ might increase substantially depending on the persistent infectious tail length $v t^{\prime}$.

\subsection{Overview of the simulation}

We consider a system (Fig. 3) of $N$ agents moving continuously in a $2 \mathrm{D}$ arena of size $L \times L$. To study the dynamics in a quasi-continuous BMD framework,we divide unit time in $\frac{1}{\mathrm{~d} t}$ steps to study the motion of each agent. The agents are self-propelled with an average velocity of $v$ and the arena has a reflective boundary. Agents or particles move freely inside the arena, and encounter elastic collisions with themselves as well as the wall. After each collision, the direction of particle movement gets changed according to the standard rule to ensure reorientation at regular intervals.

Here every active agent or particle has one of the three kind of health status based on SIR model: Susceptible, Infected, Recovered. For the purpose of getting an overview of local infection spread, we have initialized (c)

tact causing spread of the infection to the healthy agent. c The new infected particle acts as an infection spreader independently. The infected particles will be recovered from the disease after a certain time $(\tau)$

with only one individual infected agent $\left(I_{0}=1\right)$ for every simulation. Infected agents can spread the disease in two different ways as per the assumptions of the model:

- Direct infection: A close contact (collision in terms of BMD) between any infected and susceptible person will change the active status of the healthy person into 'infected' making them infectious to others.

- Indirect infection: Other than the direct contacts, the effect of indirect infections are also taken under consideration in this model (Fig. 4). An infectious tail propagating along with every active infected agent/particle is considered, following the arguments discussed in Sect. 2.1. This tail signifies the formation of infectious puff cloud and surface contamination effect. The length of this infectious tail is set up to $\delta$ steps relating to the particle's past positions in the simulation. Initially these parameters (infectious tail length, speed of the particles) are set to a certain value on the simulation. The extent of the tail could be related to the duration of the activeness of fomite or surface contamination effect. Any particle having 'susceptible' status, passing through this tail will alter its health status to 'infected'.

- There is no difference, in terms of the infection spreading, between these indirectly infected individuals and those who got infection of direct kind. Both these infected agents are capable of transmitting the disease to other susceptible individuals.

On average, after a certain amount of time $\tau$ after getting the infection, known as 'recovery time', the status of infectious person will change into 'recovered', to keep track of recovery (and full immunity from the disease) of that individual. After that, the alteration of the health status of that particular agent would not be possible. 


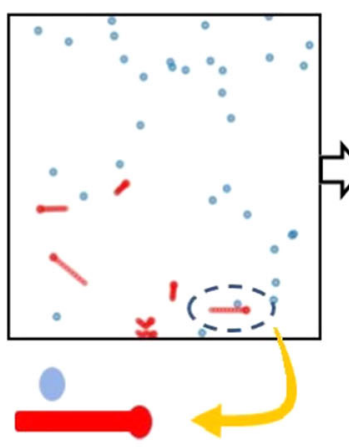

(a)

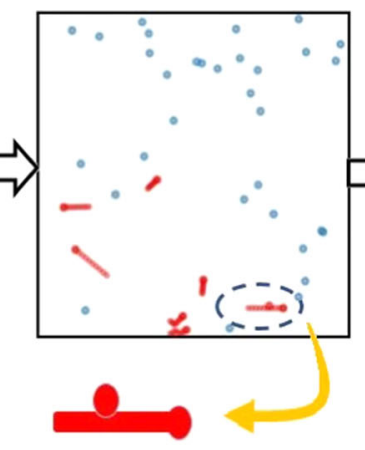

(b)
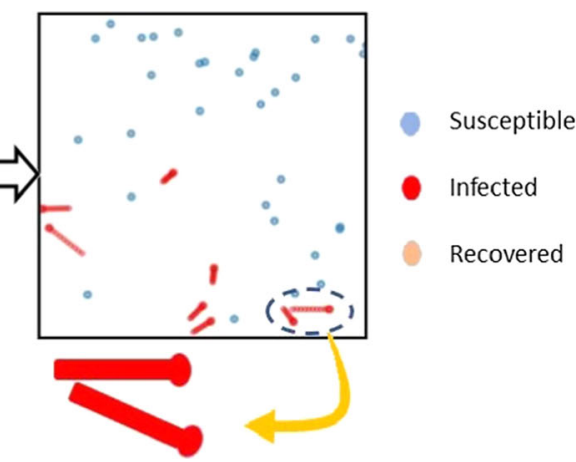

(c)
Fig. 4 A series of snapshots from the simulation considering self propelled particles with direct as well as indirect infections. a The simulation arena of size $1.5 \times 1.5$. $N$ active, self propelled, with infectious tail particle present. b A close contact to the infectious tail causing spread of the infection to the healthy agent. c The new infected particle acts as an infection spreader independently. A video depicting the dynamics of direct infection and a dynamics containing both direct and indirect infections can be found at https://youtu. be/wHVBCEX5RB0

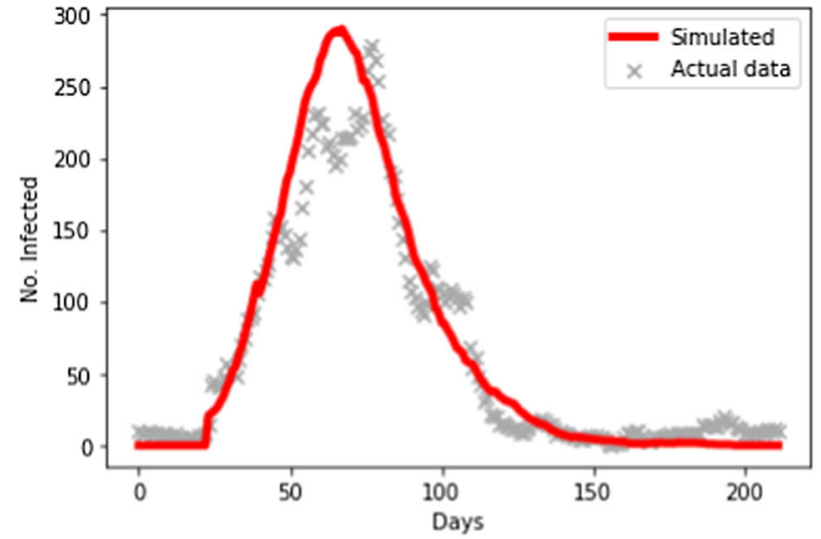

(a)

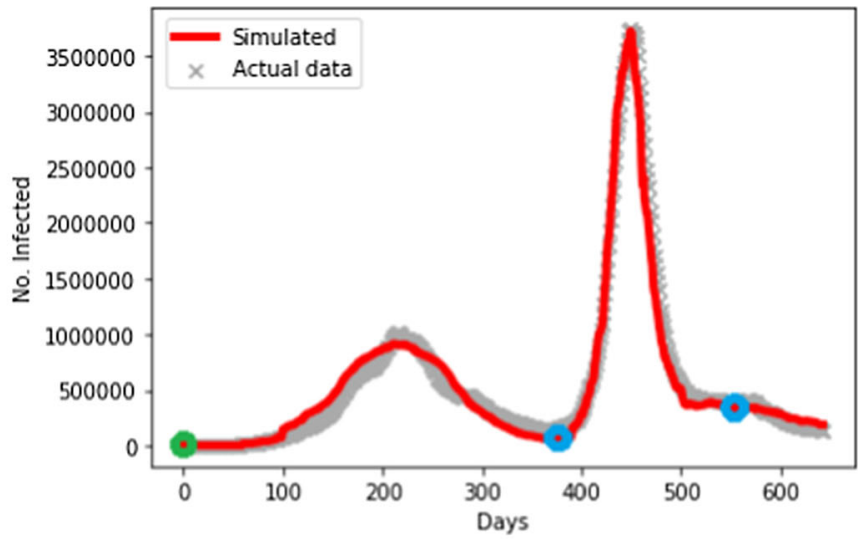

(b)

Fig. 5 Model validation with real data: a Fitting of localized active cases in a small geographic region, Andaman \& Nicobar island. b Fitting of active cases in a large geographic region, for entire country, India

\section{Results}

\subsection{Model validation for multiple waves}

We now study the contagion process of the proposed model in a system of self-propelled agents that follow the dynamics described above. We begin by studying single-peak as well as multi-peak COVID-19 statistics in the light of our model for validation purposes. This will not only help us to estimate the model parameters associated with consecutive waves of the disease, but also establish the strength of the model in understanding real epidemic data. We wished to implement the model for both localized and large-scale infection spread. Thus, for this study, we have chosen two sets of COVID-19 infection data: (a) the case of A\&N Islands, India, and (b) the scenario of India, as a whole.

Local Level Infection Spreading (A\&N Islands): Andaman and Nicobar Islands, India, is an island state with population of 0.4 million inhabitants, which got practically sealed during the pandemic, as all ship and air travel gateways got closed due to lockdown in India. This provides us a perfect scenario to study local infection spread in a closed population. The results for the data $^{1}$ of A\&N Islands, fitted with our model is shown in Fig. 5a. In this figure, we have shown how our approach can successfully model the active cases of a comparatively smaller geographic location for a single wave, despite of the sparsity of population and inherent noisiness of active cases data for the mentioned island state.

Infection spread in Larger scale (Data for India): Next, we see the effectiveness for the model in interpreting large scale data. We chose the data ${ }^{1}$ for the entire country, India, for this validation. To handle multiple waves, we consider the active cases for the duration January 1, 2020 to November 20, 2021. We decide that the first wave starts when the active cases first becomes nonzero, and any of wave ends if the number of active people drops below $10 \%$ of the maximum active cases in that wave. Any further rise of the active cases is considered to be part of the next wave.

\footnotetext{
1 Collected from https://prsindia.org/covid-19/cases.
} 
Table 1 Multiwave epidemic data understanding: infection parameters for the consecutive waves

\begin{tabular}{lllll}
\hline & Level of infection & $\tau$ & $v$ & $\delta$ \\
\hline 1st wave & Prominent & 1.1 & 0.6 & 27 \\
2nd wave & Prominent & 0.27 & 4 & 45 \\
3rd wave & Less significant & 1.1 & 1 & 15 \\
\hline
\end{tabular}

In Fig. 5b, the green circle indicates the starting of the first wave, and the blue circle indicates the end of a particular wave and beginning of the next wave. As can be seen in Fig. 5b, the model performed reasonably well in fitting the data with multiple partially overlapping waves. The parameters for all consecutive waves are presented in Table 1 . It can be seen that the parameters vary significantly in different waves which might be because of different level of people mobility, virus variants and their contamination strengths.

We note that for both local and global levels, the parameters for individual waves can indicate relevance of different physical aspects. The major factors or parameters that affect the dynamics are mentioned in Table 2. These parameters can have prominent effect on the epidemic characteristics. The reliability of the model in data interpretation shows that different types of disease outbreaks could be connected to the proposed framework by setting up these parameters appropriately. Especially, in the diseases where fomite infections can have significant effects, there the proposed model gives flexibility in understanding the infection process, incorporating possibilities of different virus strains. Thus, by varying these epidemic parameters, we will get a clear understanding about the mechanism of different infection outbreaks.
Thus, we now particularly investigate the influence of different epidemic parameters, one by one, on the dynamics of self-propelled particle population through the simulations. This would help us to get a clear knowledge about the efficacy of the epidemic parameters. At first, the analysis of the results from the BMD based simulation with different tail lengths is done. Then, we have analyzed the model by changing the population inside the arena. Recovery time of any infected individual has a significant role for any kind of epidemic. It determines for how much of time it will spread the infection through direct or indirect contact in a susceptible population. Considering this case, we have also varied the recovery time on different simulations. Finally, the speed of the active agents inside the arena is varied. Analysis of the simulation results with this scheme have been studied in following sections.

\subsection{Effect of indirect infection tail}

We begin our analysis by varying one of the key dynamical parameters, the length of infectious tail, $\delta$, and fixing the other epidemic parameters for each simulation. We fix the size of the arena to be $L=1.5$, and this size is kept unaltered in most of the simulations of this study, unless mentioned otherwise. In Fig. 6a the effect of the infectious tail or indirect surface infection has been depicted. The dynamics is studied with tail length of 0,8 and 15 . To quantify our understanding further, we define a couple of quantities that relate to some key characteristics of the dynamics. The first such quantity, Epidemic size $I_{\text {total }}$, is the total fraction of people who get infected during the entire course of the disease. This is the number of people who got infected during the course of the epidemic and it estimates the extent at which the disease affected the population. It is observed that $I_{\text {total }}$ is strongly dependent

Table 2 Epidemiological parameters for direct and indirect infection

\begin{tabular}{|c|c|c|c|}
\hline Parameters & Notation & Physical relevance & Range used in study \\
\hline Population number & $N$ & $\begin{array}{l}\text { Density of Population } \\
\text { in a locality }\end{array}$ & $10-200$ \\
\hline Tail length & $\delta$ & $\begin{array}{l}\text { Survival of Surface and } \\
\text { puff cloud contamina- } \\
\text { tion. Depends on differ- } \\
\text { ent virus strains }\end{array}$ & $0-15$ \\
\hline Recovery time & $\tau$ & $\begin{array}{l}\text { Duration of infectious } \\
\text { period of an infected } \\
\text { individual over the } \\
\text { epidemic. Depends on } \\
\text { health infrastructure } \\
\text { and different virus } \\
\text { strains. }\end{array}$ & $0.1-1.5$ \\
\hline Particle speed & $v$ & $\begin{array}{l}\text { Movement of the } \\
\text { infected individual. } \\
\text { Depicts level of aware- } \\
\text { ness, precautions and } \\
\text { restrictions }\end{array}$ & $0.5-2.0$ \\
\hline
\end{tabular}




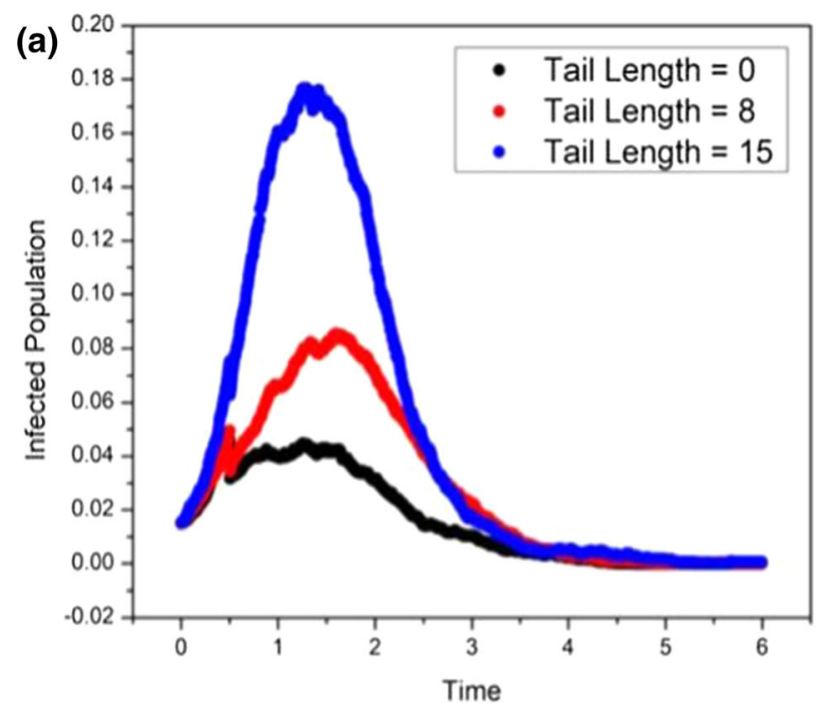

Fig. 6 Indirect infections and infectious tail: a Simulation data (averaged 100 times) by varying infectious tail length of the infected agents inside the arena. $N=70, \tau=0.5, v$ $=1.0, \delta=0,8$, and 15; b Exploration of fraction of max-

on tail length: from $20 \%$ for $\delta=0$, it rises to $34.3 \%$ for $\delta=8$ and $60 \%$ for $\delta=15$. It is clearly understandable through the increasing size of the bell-shaped curves that a longer tail generates notably more number of new infections among the population than a withouttail individual. It shows that, in the diseases where the indirect infections play an important role in the dynamics of disease spreading, the outcome of infected fraction can get affected significantly resulting into a substantial fractional increase. These results clearly highlight the importance of accommodating the necessity of the 'tailed' agents in the model. Here we must mention that, the little notch-like fall in infected population at $T=0.5$ is due to the fact that, the initial infected particle gets recovered in that certain time for every simulation over 100 times.

The height of infected distribution peak or fraction of maximum infection, $I_{\max }$ serves as another important quantity to explore. We have analyzed our model by exploring the epidemic peak for each case as the number of infected individuals at a particular time-point directly tests the available health facilities causing a lack of basic supporting infrastructures, like, medical oxygen supply. Thus, we study the effects of persistent indirect infection tail on $I_{\max }$. We consider different values of $\delta$ to incorporate how long a particular infection persists on a surface, with all the other parameters fixed at $N=70, \tau=0.5, v=1.0$. As shown in Fig. 6b, tail length of the infected agents influences the $I_{\max }$ considerably. It starts growing up significantly after a certain value of $\delta=6$ for the moderate population density we have considered.

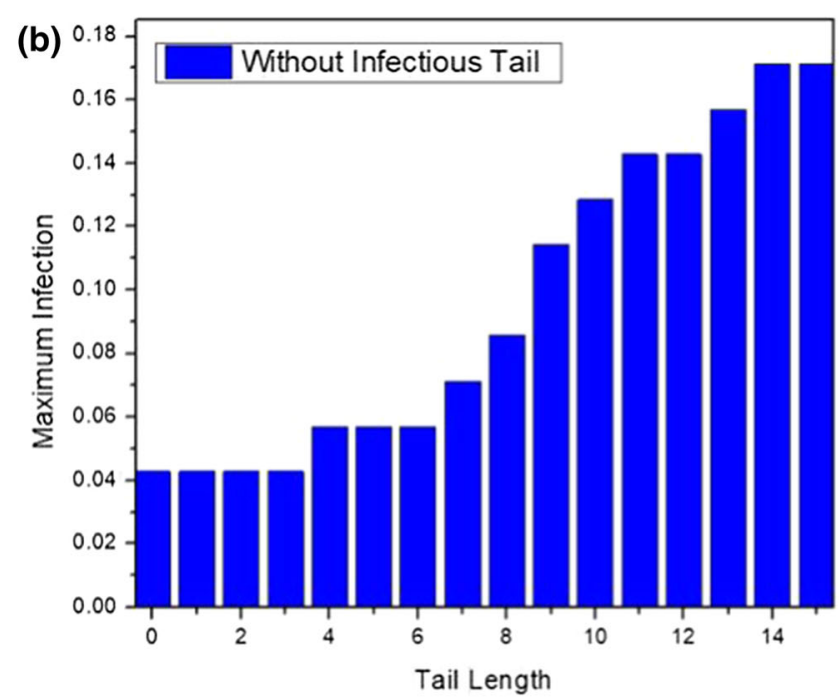

imum infection, $I_{\max }$ with varying tail length of infectious particles in the artificial society. The other parameters are $N=70, \tau=0.5, v=1.0$

\subsection{Effect of population density}

Population density works as a major controlling factor when a disease spreads from person to person. Thus, for the diseases with direct as well as indirect infection, we study the effect of population density. This particular investigation also explores the infection spreading process separately in a low and dense population regions. In Fig. 7a, b, we compare a set of simulations on our synthetic society. Figure $7 \mathrm{a}$, b exhibit the average results of the dynamics for $N=70$ and $N=180$ respectively. In both the figures red (blue) color signifies dynamics with (without) indirect infection; in terms of parameters, two values $\delta=8$, and 0 were chosen to incorporate the presence or absence of infectious tail. Other parameters are fixed at $\tau=0.5$ and $v=1.0$.

The results show significant impact of the population density on the infection dynamics. For $N=70$ (Fig. 7a) the infectious tail has a prominent effect in infection spread among the population and $I_{\max }$ increases more than twofold as the indirect infection begins to spread. A substantial rise in the epidemic size is also noted as we observe $\sim 15 \%$ increment in total infected population for $\delta=8$ when $N=70$. It is also noticeable that in case of very dense population (Fig. 7b), the probability of getting direct infection is very high. The motion of the particles become very much interactive through several collisions on their way. So, spreading of new infections through indirect contacts (infectious tail) is not a significant factor here. Hence, the difference between 'with tail' and 'without tail' simulation is also negligible in this case. However, we must note 

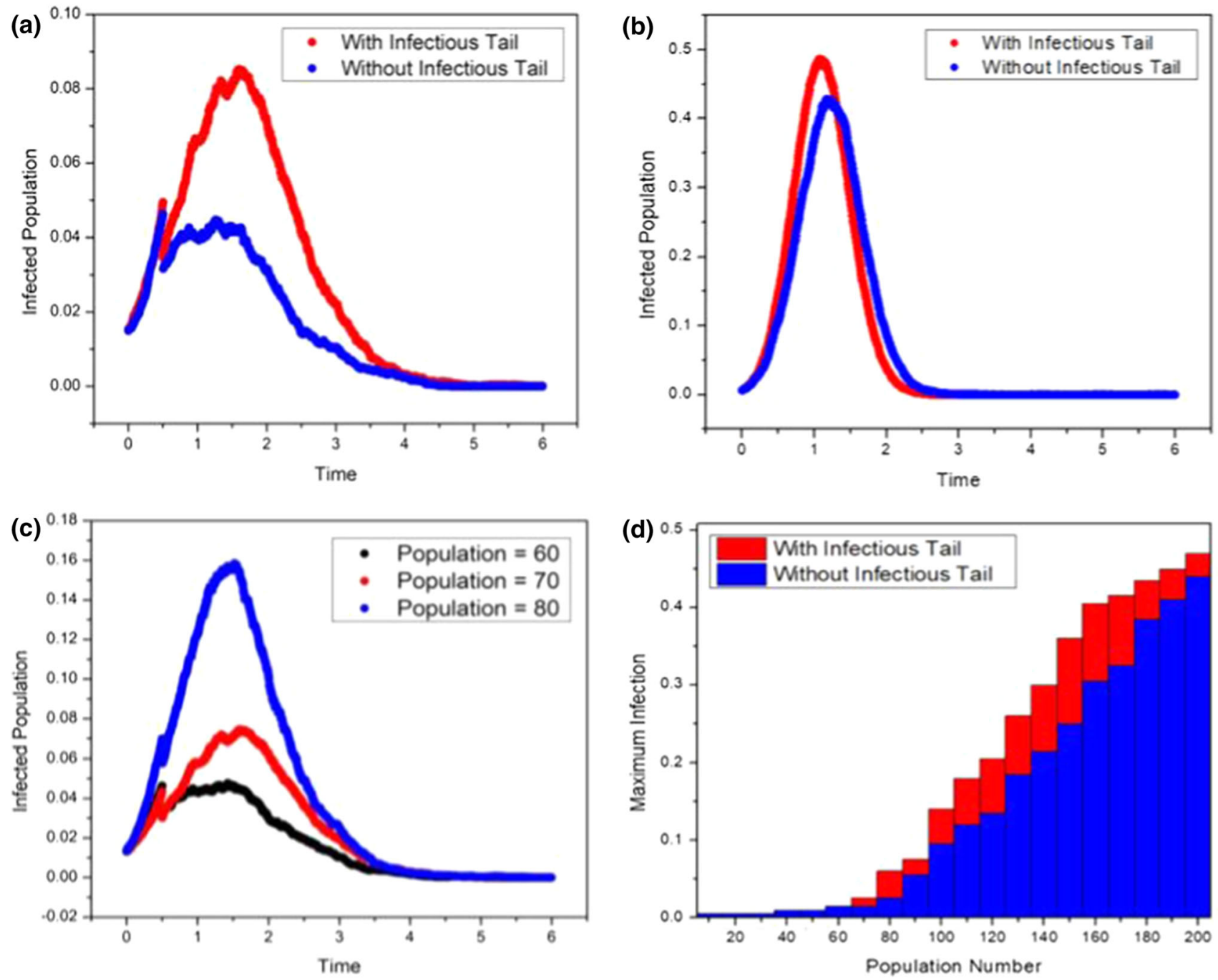

Fig. 7 Indirect infections and population density: a Comparison between the simulation data (averaged 100 times) of both cases ('with infectious tail' and 'without tail') for moderate number of population inside the arena. Chosen parameters are $N=70, \tau=0.5, v=1.0, \delta=8$ (with tail) and, 0 (without tail). b Comparison between the simulation data (averaged 100 times) of both cases ("with infectious tail' and 'without tail') for highly dense population inside the arena. Parameters are $N=180, \tau=0.5, v=1.0, \delta=$

that though the cause of the indirect infection loses its importance here, the rise of infection is very sharp and rapid.

Simulation outcome by varying population inside the arena $N$ is shown in Fig. 7c. For these particular simulations, $N$ was chosen as 60,70 and, 80 , respectively. The results show that the fraction of infected individuals considerably increase as the population density grows gradually. The increasing population in a fixed area will enhance the chance of getting infected. The compactness of the arena generates highly interactive and complex motion in the population. Thus, the infection probability increases with the increasing popula-

8 (with tail) and, 0 (without tail) respectively. c Simulation data (averaged 100 times) by varying population number in the arena. $N=60,70$ and, 80, $\tau=0.5, v=1.0, \delta=8$. d Comparison between the simulation results of both cases ('with infectious tail' and 'without tail') by exploring the point of maximum infection, $I_{\max }$ with varying number of population in the artificial society. The other parameters are $\tau=0.5, v=1.0, \delta=8$ and, 0

tion density inside the arena. Hence, the number of infected population increases drastically. The maximum infection fraction, $I_{\max }$ also has a considerable dependence on the population density. In Fig. 7d, we explore this observation systematically. Here, height of the red (blue) bars signify maximum infective fraction, $I_{\max }$ for the dynamics with (without) indirect infections. The rapid increase in this value is noticed after a threshold point of $N=60$. The difference between two type of cases('with tail' and 'without tail') is clearly visible from the results. It shows the importance of taking indirect infections into account for cases with moderate population density. 
However, for very low and very high population density, the contribution of the indirect infections through the tail is nearly negligible. We have already discussed the possible reasons behind the observations for high population density; in case of less population density, the chance of both type of contacts (direct and indirect) is very less. Thus, the difference between the results of 'with' and 'without infectious tail' simulation is negligible in low population density. For a random agent, there are so much space to move inside the arena that the role of the infectious tail also became insignificant.

\subsection{Effect of recovery time}

Recovery time of the infected agents could be a critical parameter for infectious disease spreading dynamics. An infected individual with long recovery time basically contributes in increasing the Reproduction number, $\mathcal{R}$; reproduction number has a standard definition as the number of infected people generated by a single infectious person in his entire infectious lifetime. In this section, we try to understand what is the effect of indirect infections due to surface fomites in the context of diseases with different recovery rates.

In the presence and absence of infectious tails, we study the system dynamics for two different recovery rates (Fig. 8a, b). In case of relatively lower recovery time $\tau=0.5$, Fig. 8a, the difference in total infected number is significantly different; $I_{\text {total }}$ rises to $34.3 \%$ $(\delta=8)$ from $20 \%(\delta=0)$ as infectious tails are introduced. As recovery time is increased to $\tau=1.0$, in Fig. $8 \mathrm{~b}, I_{\text {total }}$ increases drastically. The difference between 'with tail' and 'without tail' simulation result is also illustrated in this figure, for higher recovery time. In case of 'with infectious tail' particles, the number of new infection generated is higher, and thus the infection spread occurs in a much faster rate. However, the time for its recovery clock is also started a little early for these particles, resulting into a sharp and long shaped curve. For 'without tail' though the spread of the infection occurs in a relatively slow rate, the recovery also occurs gradually, giving rise to a relatively long-tailed distribution. Thus, the width is slightly broader in this particular case and the total number of infected population is not much different $\left(I_{\text {total }}=91.4 \%\right.$ for $\delta=8$ and, $87.1 \%$ for $\delta=0$ ).

We consider the effect of recovery rates on the dynamics for a fixed tail length next. Figure $8 \mathrm{c}$ shown the result for Infected population distribution for $\tau=$ $0.5,1.0$ and, 1.5. The other parameters are $N=70, v$ $=1.0, \delta=8$. We note that as the recovery time gets higher, the bell-shaped curves rise drastically. Results show that $I_{\text {total }}$ changes from a mere $34.7 \%(\tau=0.5)$ to a massive value of $91.4 \%(\tau=1.0)$ and $100 \%(\tau=1.5)$ respectively. Thus, we observe that the higher recovery time of the infected agents could be very crucial and results into a devastating epidemic size.

Along with the increase in epidemic size and reproduction number, we also note that the maximum infection fraction, $I_{\max }$ increases and also shifts towards right with higher recovery times. We study this behavior more closely in Fig. 8d. In similar bar charts as before, we study 'with' (red) and 'without tail' (blue) scenarios in the context of maximum infection. We observe a threshold point ( $\tau=0.4$ for this parameter settings), beyond which the effect of indirect infections of $I_{\max }$ increases very fast as recovery rates grow. Here, the difference between the two cases maintained nearly equal interval along the whole range of recovery times, beyond the threshold. However, for very low recovery time, both of the two cases shows similarity in maximum infection values, due to fast recovery and less infectious lifetime, affecting both direct and indirect spreading. Thus, from the overall study, we conclude that for moderate to high recovery times, indirect infections can cause substantial differences in terms of both $I_{\text {total }}$ and $I_{\max }$.

\subsection{Effect of rapid movements}

Another important criteria for epidemic spread is the movement of agents involved. In this section, we study the coupled effect of indirect infections and speed of movement of the agents. We study this effect by modulating the motion of the particles inside the arena. Fast movement among the population signifies the restriction-free long travel of infected persons after epidemic outbreak. On the other hand, a very less average velocity of the particles signifies high precautions and travel restrictions. Here, a particular infected person is constrained to move only in a localized area.

The results from Fig. 9a, b compare the effect of the indirect infection in the context of speed of the particle. Depicted in Fig. 9a, is the scenario with $v=1.0$ that can be considered as a normal scenario. Speeding up the agents would be if $v$ is increased beyond 1 , while reducing $v$ below 1 signifies movements are being slowed down due to imposed government regulations. For the high speed particles the infection spread occurs in a fast rate, as shown in Fig. 9b. The initial infected individual in the simulation spreads the infection rapidly with more number of collisions within his total infection period. Whereas, in moderate movement of the particles, the infected ones do not get enough time to execute longer trajectories among the susceptible population. Thus, the chance of getting a close contact with other particles became remain moderate. We see a drastic jump in the total infection size from $20 \%$ to $\sim 73 \%$ as $v$ increases from 1.0 to 1.5 , even without the presence of any indirect infection. In presence of infectious tails, instead of $34.7 \%$ (for $v=1.0$ ), $87 \%$ get infected in the artificial society considered. The simulation results with slow speed particles signifies the importance of high precautions and restrictions among the population. It is clearly visible that the spread of the infection is under control in this situation.

Figure 9c exhibits the effects of movement as well as speed for a constant tail length. An increase in the average speed of the particles inside the arena has affected the variance and height of the infected population distribution, making the bell-shaped curve taller and nar- 

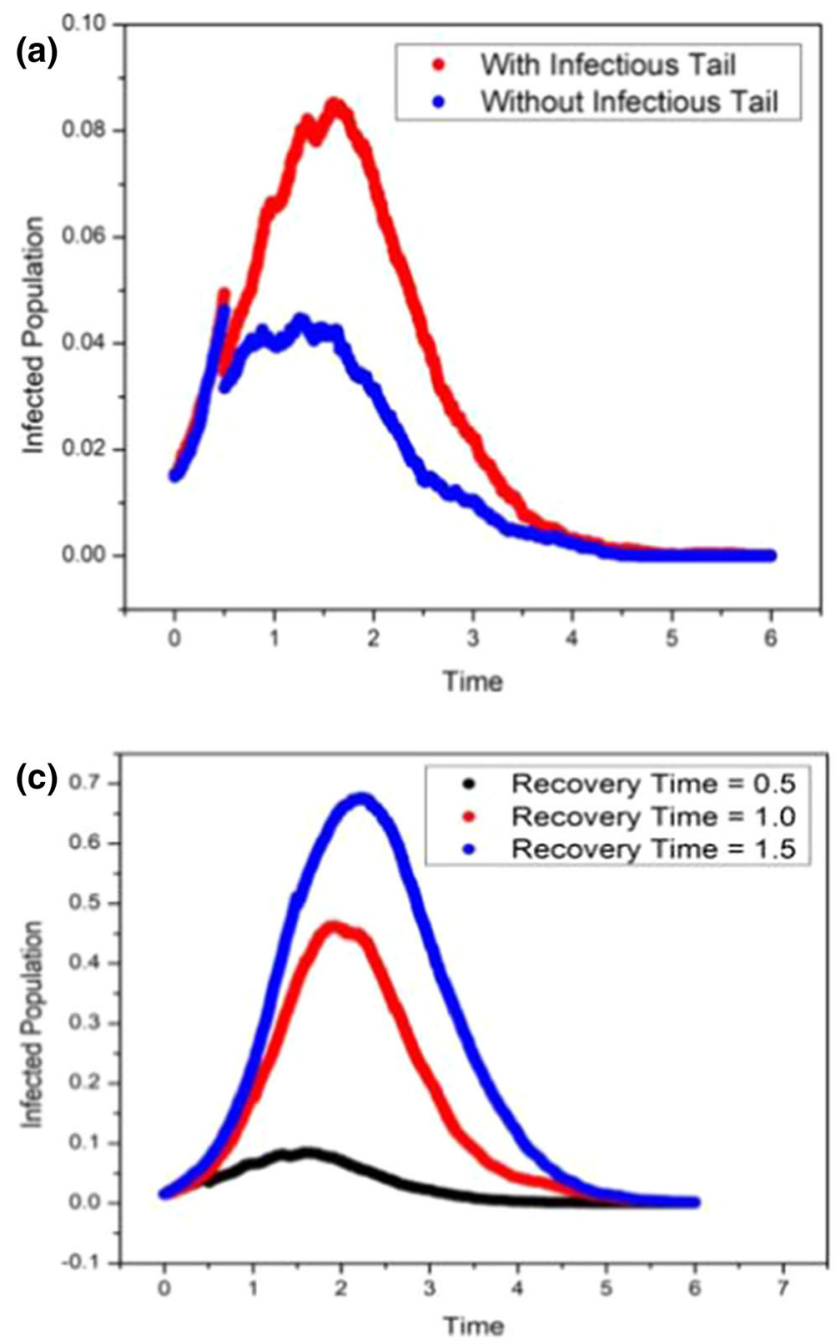

Fig. 8 Indirect infections and recovery time of the infected: a Comparison between the simulation data (averaged 100 times) of both cases ('with infectious tail' and 'without tail') for moderate recovery time of infected individuals of the society. Parameters are $N=70, \tau=0.5$, $v=1.0, \delta=8$ (with tail) and, 0 (without tail). b Comparison between the simulation data (averaged 100 times) of both cases ('with infectious tail' and 'without tail') for relatively higher recovery time of infected individuals of the

rower. Moreover, the epidemic size also changed from 34 to $95 \%$ when average speed is made double. This is due to the fact that the fast the movement of the particles cause frequently occurring interactions with the susceptible particles, making then come into the infectious range. But, as the infections is initiated in a faster rate, the recovery clock for the infected agents also starts earlier. So, although there would be a rapid increase in infections, the recovery takes dominance earlier as well, resulting to a high and sharp peak in infected fractions. These kind of distributions put direct pressure on available health infrastructure causing a rapid demand of life-supporting facilities for a large number of infected people. The significance of the speed in the simulation
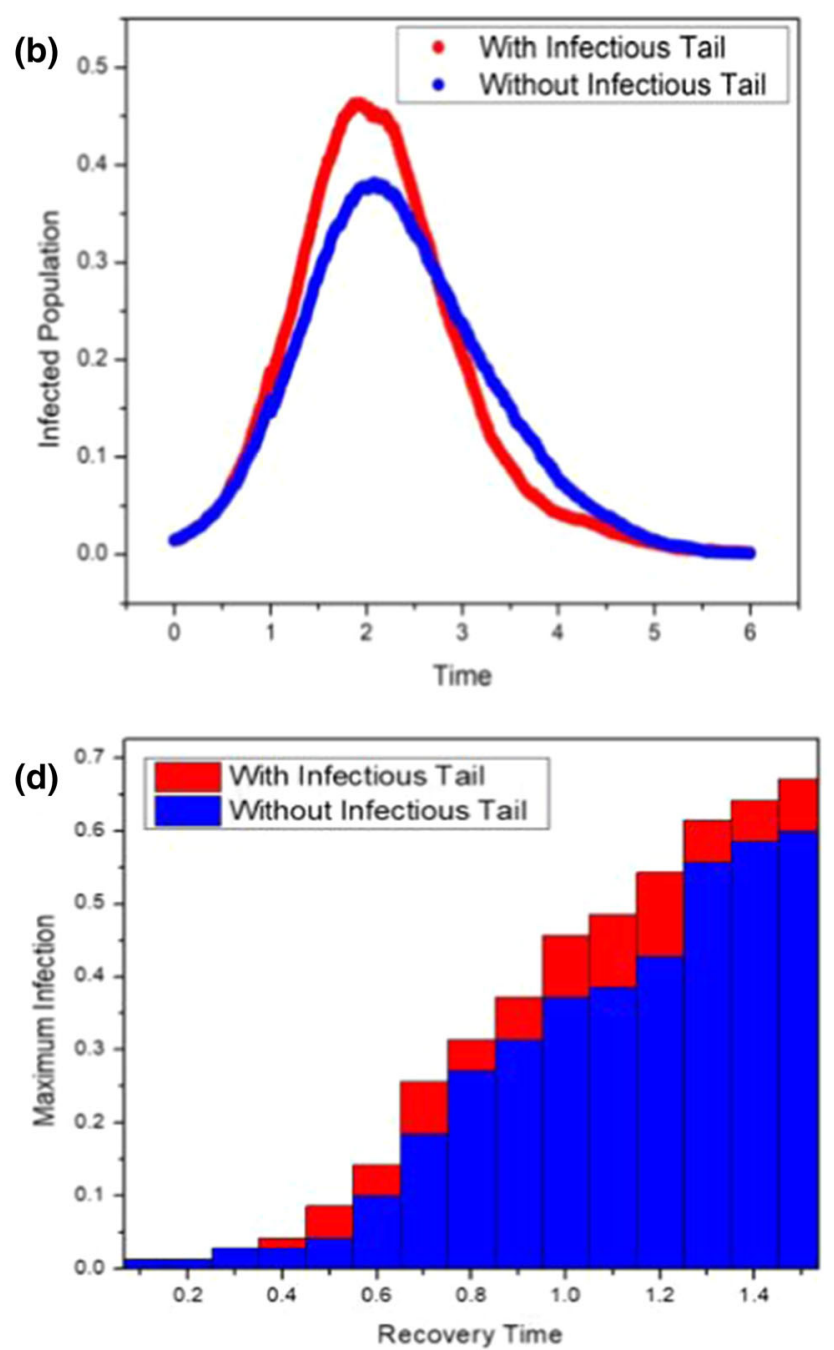

society. Parameters are $N=70, \tau=1.0, v=1.0, \delta=8$ (with tail) and, 0 (without tail). c Simulation data (averaged 100 times) by varying recover time for infected agents inside the box. Parameters are $N=70, \tau=0.5,1.0$ and, 1.5, $v=1.0$, $\delta=8$. d Comparison between the simulation results of both cases ('with infectious tail' and 'without tail') by exploring the 'point of maximum infection' with varying recovery time of the infected agents. $N=70, \tau=$ varying, $v=1.0, \delta=8$ (with tail) and, 0 (without tail)

could be directly related to the level of imposed travel restrictions and precautions.

By increasing the average speed of the system, the value of $I_{\max }$ starts growing in number. We report the results in Fig. 9d. For relatively slower speed, i.e., very strong movement restrictions of particles, the difference between two type of cases is ignorable, showing that regulations will control the disease even in presence of indirect infections. However, the differences increases immediately for moderate to high speed movement of the agents. Beyond a threshold speed $(v=0.8$ in the case of this particular set of observations) the difference becomes notably larger. The prominent differences between heights of the with and without tail bars (red 

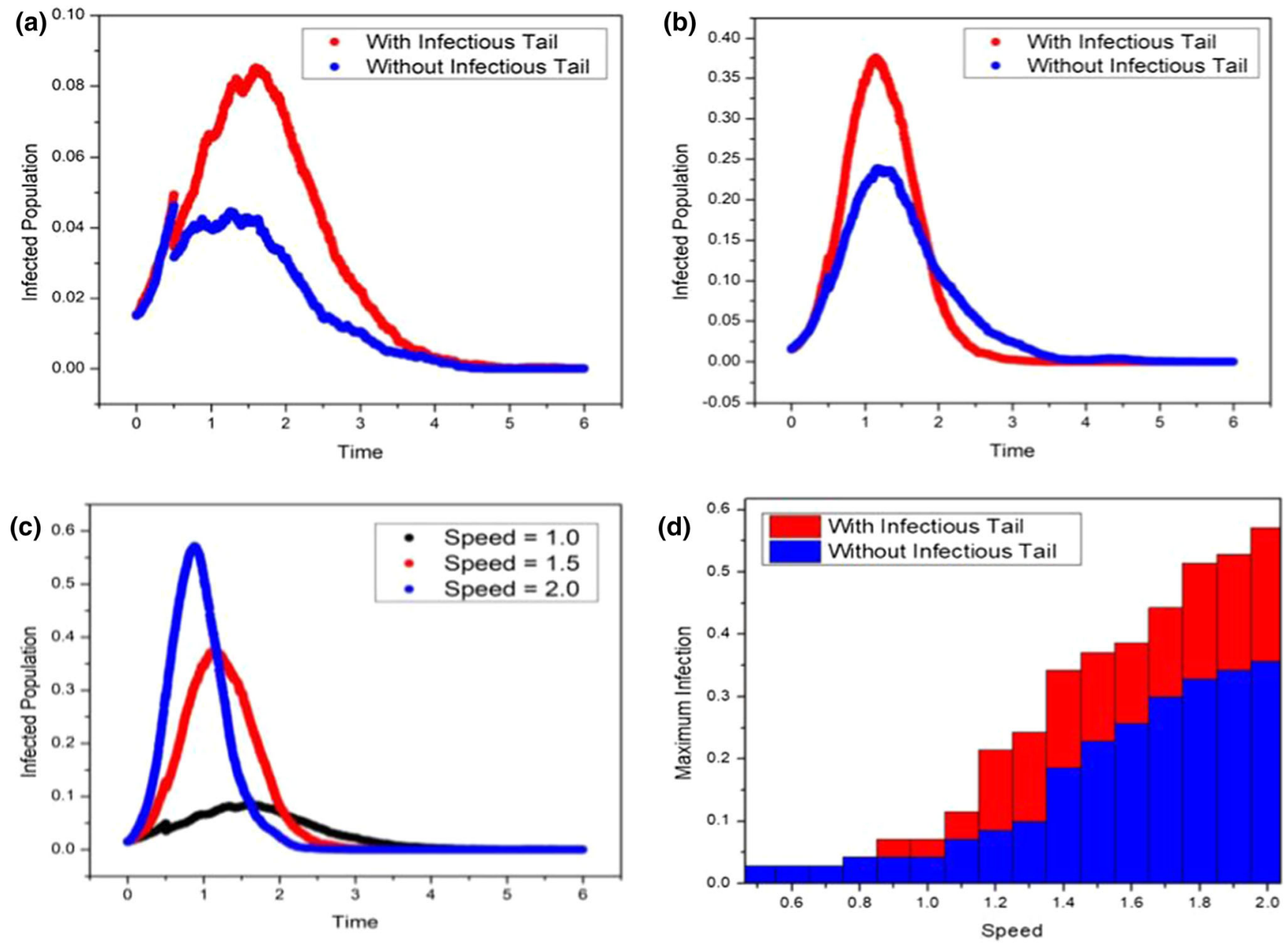

Fig. 9 Indirect infections and Speed of movement: a Comparison between the simulation data (averaged 100 times) of both cases ('with infectious tail' and 'without tail') for moderate speed of particles inside the arena. $N=70, \tau=0.5$, $v=1.0, \delta=8$ and 0 . b Comparison between the simulation data (averaged 100 times) of both cases ('with infectious tail' and 'without tail') for faster motion of particles inside the arena. $N=70, \tau=0.5, v=1.5, \delta=8$ and, 0 .

and blue respectively) in the figure, establish the importance of considering the indirect contamination in the dynamical models, wherever movements are not absolutely restricted.

\subsection{Correct interpretation of data: the context of indirect infections}

In the previous sections, we have thoroughly described the coupled response that can get created due to interplay of epidemic parameters with the indirect infections. We also assigned physical relevance to each of our model parameters. In this section, we demonstrate the necessity to consider indirect infections, wherever applicable, in the context of data interpretation as well. Computational study of any artificial society and the dynamics within, has a final goal of relating, interpret-

c Simulation data (averaged 100 times) by varying speed of the particles inside the arena. $N=70, \tau=0.5, v=1.0$, 1.5 and, $2.0 \delta=8$. d Comparison between the simulation results of both cases ('with infectious tail' and 'without tail') by exploring the 'point of maximum infection' with varying average speed of the particle motion. $N=70, \tau=0.5, v=$ varying, $\delta=8$ and 0

ing and predicting real data, by accurate estimation of model parameters. Important thresholds associating the model parameters help to understand the disease outbreak and, at the same time, can be exploited as control strategies. Here, we study how the parameter estimation for a dynamics can have flaws if indirect infections are not considered. For illustration, we have considered a model data for infection spread. The data represents a general infectious disease, where the fraction of infected people has risen rapidly during the epidemic period. It reaches a peak (maximum infection) and then gradually falls towards zero (or insignificant) number of infections with time. Though we have chosen this synthetic data to highlight our observations, we must point out that the related results and discussions are very much general, in the context of the framework, and are applicable to any infection spreading data, subjected to interpretation. 


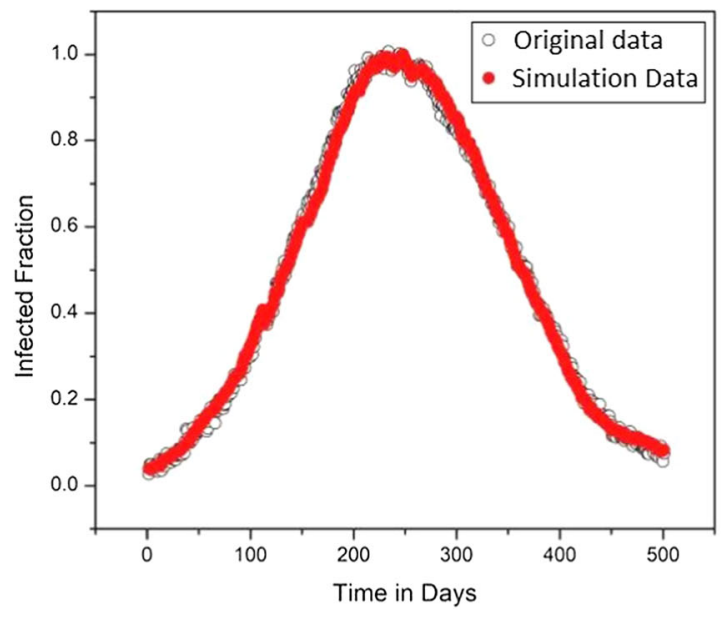

(a)

Fig. 10 Parameter estimation from data: a (and b) respectively depict comparison of epidemic data fitted with simulation data (averaged 100 times) for active agents 'with (and without) infectious tail'. For $N=70, \tau=1.1$. Other

We have divided our investigation in two cases and shown the results in Fig. 10. We have considered both the possibilities of the epidemic spread: first, through direct interactions, and then, including indirect infections from infectious tail with the previous. Here, for these data, let us consider the fact that indirect infections were important for this spreading process. However, while analysis, we may have not included the possibility in the simulations. Thus, in the first case, the data are fitted with the simulation results of 'without infectious tail' agents. Then, a comparison is performed considering 'with infectious tail' simulation for fitting the synthetic epidemic data. We have fitted these curves to get an intuitive idea about the difference in epidemic dynamics parameters and the control strategies.

To exhibit the comparative view, we have used the normalized data. In Fig. 10a, 'without infectious tail' simulation outcomes are fitted to the data by setting up the epidemic parameters carefully to $N=70, \tau=1.1$, $v=0.85, \delta=0$. In Fig. 10b, to fit the same data with 'with infectious tail' case, we have a different set of parameter values, $N=70, \tau=1.1, v=0.6, \delta=25$. While the goodness of fit of both these scenarios are explicitly visible in the figures, we hereby highlight the striking difference in the velocity parameter value. It indicates that while we are not considering the indirect infection, it seems like the travel restrictions protocols implemented due to the outbreak of the epidemic are not getting followed properly and there is only $\sim 15 \%$ reduction in movement $(v=0.85)$ compared to the normal case $(v=1)$. However, while we consider the indirect infection, it is evident that similar infection spreading statistics may come even with $\sim 40 \%$ reduction of the movements $(v=0.6)$ compared to the normal scenario. This contrasting value of $\delta$, might also relate to a new virus strain which survives for a longer period of time on surfaces, causing a more persistent 'tail'. As

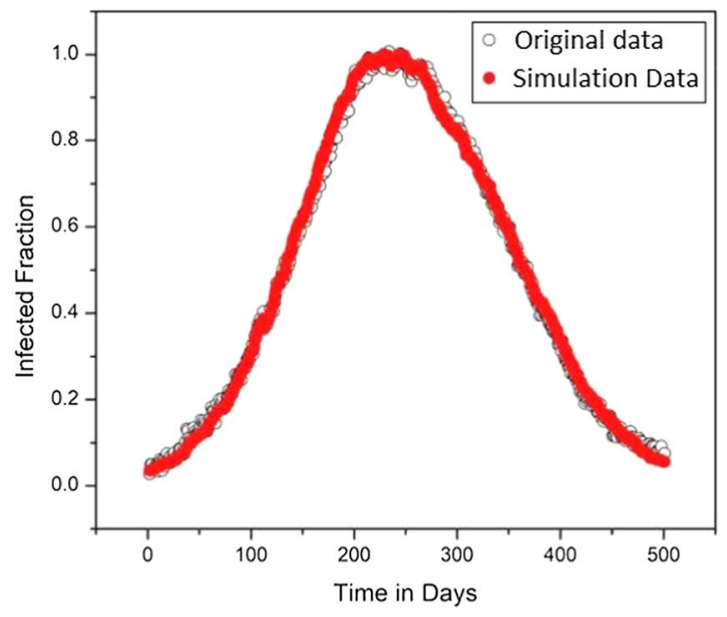

(b)

parameters are found to be different for the same data: in a $v=0.85, \delta=0, \mathbf{b} v=0.6, \delta=25$ However, epidemic size, $I_{\text {total }}$ is almost same in both cases, $78-80 \%$ agents for both the simulation data

demonstrated in Fig. 10b, though the outcome might indicate that the that travel restrictions are being violated, the indirect infection can increase the infection count even in the presence of imposed restrictions and lockdown in reality. Thus, in the absence of indirect infection in the model, comparatively larger infection rates and relaxation of restrictions can be estimated that may fail to capture the reality of the situation.

\section{Conclusions and future works}

Worldwide spread of COVID-19 has established how fast an infectious disease can become a pandemic and can cause a major blow to humanity. The spreading process of these diseases are majorly considered as a direct transmission in most of the recent mathematical and computational models. However, the possibility of transmission through contaminated surfaces or objects are often ignored. In this paper, we demonstrated the impact of indirect infection spreading dynamics on overall model parameter estimations and their understandings. The proposed methodology has been tailormade to accommodate the possibilities of direct as well as indirect ways of infection propagation. We validated the model using active case data of COVID-19 infection and demonstrated that this framework can be successfully applied for single as well as multiwave epidemic data. We methodically explored the contributions of the key parameters, like population density, recovery rate, rapid movements on the epidemic dynamics, and exhibited the drastic differences that may appear in the presence of the indirect infection. As shown in our experiments, the effect of indirect infection is vivid when the population density is moderate, which is in fact the case in most of the counties in the world. The interplay 
of indirect infections with important epidemic parameters like recovery rate and movements of individuals have been explored as well, giving rise to insightful observations. The striking differences between the cases where the indirect infections are considered compared to the cases where only the direct infection is considered made it clear that while modeling an infectious disease, we need to be more careful and thorough. Exhibiting ambiguity in parameters while data interpretations, we have established that overlooking the indirect infection might lead to erroneous parameter estimation. This can prevent us to understand the real dynamics of the pandemic and may restrict us to design optimal strategies.

In this paper, we demonstrated the importance of the indirect infection assuming three health statuses: susceptible, infected and recovered with simplistic contagion rules. However, in future, the idea can be extended to other epidemic models considering contact tracing, quarantining, vaccination and deceased subpopulations. Straightforward extensions of this framework can be used to practically seal the neighborhood of a fraction of infected individuals, making them inaccessible to the susceptible particles, and thus incorporating quarantine. From susceptible state, a fraction of active particles can take a direct transition to immune state and remain unaffected by the infectious people and surfaces, which will indicate vaccination. Analyzing all these complex dynamical scenarios under the light of indirect infection will give us an holistic picture of the pandemic.

\section{References}

1. World Health Organization et al. Transmission of sarscov-2: Implications for Infection Prevention Precautions: Scientific Brief, 09 july 2020. Technical report, World Health Organization (2020)

2. V. Stadnytskyi, C. Bax, A. Bax, P. Anfinrud, The airborne lifetime of small speech droplets and their potential importance in sars-cov-2 transmission. Proc. Natl. Acad. Sci. 117, 202006874, 05 (2020)

3. N. Singh, M. Kaur, On the airborne aspect of covid-19 coronovirus. arXiv preprint arXiv:2004.10082, (2020)

4. M. Ciotti, M. Ciccozzi, A. Terrinoni, W.-C. Jiang, C.B. Wang, S. Bernardini, The covid-19 pandemic. Crit. Rev. Clin. Lab. Sci. 57(6), 365-388 (2020)

5. R. Zhang, Y. Li, A.L. Zhang, Y. Wang, M.J. Molina, Identifying airborne transmission as the dominant route for the spread of covid-19. Proc. Natl. Acad. Sci. 117(26), 14857-14863 (2020)

6. L. Setti, F. Passarini, G. De Gennaro, P. Barbieri, M.G. Perrone, M. Borelli, J. Palmisani, A. Di Gilio, P. Piscitelli, A. Miani, Airborne transmission route of COVID-19: why 2 meters/6 feet of inter-personal distance could not be enough. Int. J. Env. Res. Pub. Health $\mathbf{1 7}(8), 2932$ (2020)

7. R. Karia, I. Gupta, H. Khandait, A. Yadav, A. Yadav, Covid-19 and its modes of transmission. SN Compr. Clin. Med. 2, 1798-1801 (2020)
8. L. Luo, D. Liu, H. Zhang, Z. Li, R. Zhen, X. Zhang, H. Xie, W. Song, J. Liu, Q. Huang et al., Air and surface contamination in non-health care settings among 641 environmental specimens of 39 covid-19 cases. PLoS Negl. Trop. Dis. 14(10), e0008570 (2020)

9. J.L. Santarpia, D.N. Rivera, V.L. Herrera, M.J. Morwitzer, H.M. Creager, G.W. Santarpia, K.K. Crown, D.M. Brett-Major, E.R. Schnaubelt, M.J. Broadhurst et al., Aerosol and surface contamination of sars-cov2 observed in quarantine and isolation care. Sci. Rep. 10(1), 1-8 (2020)

10. B. Wan, X. Zhang, D. Luo, T. Zhang, X. Chen, Y. Yao, X. Zhao, L. Lei, C. Liu, W. Zhao et al., On-site analysis of covid-19 on the surfaces in wards. Sci. Total Environ. 753, 141758 (2021)

11. P.Y. Chia, K.K. Coleman, Y.K. Tan, S. Wei Xiang Ong, M. Gum, S.K. Lau, X.F. Lim, A.S. Lim, S. Sutjipto, P.H. Lee et al., Detection of air and surface contamination by sars-cov-2 in hospital rooms of infected patients. Nat. Commun. 11(1), 1-7 (2020)

12. J. Zhou, J.A. Otter, J.R. Price, C. Cimpeanu, D. Meno Garcia, J. Kinross, P.R Boshier, S. Mason, F. Bolt, A.H. Holmes, et al., Investigating sars-cov-2 surface and air contamination in an acute healthcare setting during the peak of the covid-19 pandemic in London. Clin. Infect. Dis. 73(7), e1870-e1877 (2020)

13. G. Giordano, F. Blanchini, R. Bruno, P. Colaneri, A. Di Filippo, A. Di Matteo, M. Colaneri. Modelling the covid-19 epidemic and implementation of populationwide interventions in Italy. Nat. Med. 26(6), 855-860 (2020)

14. Z. Yang, Z. Zeng, K. Wang, S.-S. Wong, W. Liang, M. Zanin, P. Liu, X. Cao, Z. Gao, Z. Mai et al., Modified seir and ai prediction of the epidemics trend of covid-19 in china under public health interventions. J. Thorac. Dis. 12(3), 165 (2020)

15. V. Volpert, M. Banerjee, S. Petrovskii, On a quarantine model of coronavirus infection and data analysis. Math. Model. Nat. Phenom. 15, 24 (2020)

16. L. Li, Z. Yang, Z. Dang, C. Meng, J. Huang, H. Meng, D. Wang, G. Chen, J. Zhang, H. Peng et al., Propagation analysis and prediction of the covid-19. Infect. Dis. Model. 5, 282-292 (2020)

17. D. Easwaramoorthy, A. Gowrisankar, A. Manimaran, S. Nandhini, L. Rondoni, S. Banerjee, An exploration of fractal-based prognostic model and comparative analysis for second wave of covid-19 diffusion. Nonlinear Dyn. 106(2), 1375-1395 (2021)

18. C. Kavitha, A. Gowrisankar, S. Banerjee, The second and third waves in India: when will the pandemic be culminated? Eur. Phys. J. Plus 136(5), 1-12 (2021)

19. A. Gowrisankar, L. Rondoni, S. Banerjee, Can India develop herd immunity against covid-19? Eur. Phys. J. Plus 135(6), 1-9 (2020)

20. W.O. Kermack, A.G. McKendrick, A contribution to the mathematical theory of epidemics. Proc. R. Soc. Lond. Ser. A Contain. Papers Math. Phys. Character 115, 700-721 (1927)

21. C.T. Bauch, J.O. Lloyd-Smith, M.P. Coffee, A.P. Galvani. Dynamically modeling sars and other newly emerging respiratory illnesses: past, present, and future. Epidemiology 16(6), 791-801 (2005) 
22. B.M. Althouse, J. Lessler, A.A. Sall, M. Diallo, K.A. Hanley, D.M. Watts, S.C. Weaver, D.A.T. Cummings, Synchrony of sylvatic dengue isolations: a multi-host, multi-vector sir model of dengue virus transmission in senegal. PLoS Negl. Trop. Dis. 6(11), e1928 (2012)

23. A. Norambuena, F.J. Valencia, F. Guzmán-Lastra, Understanding contagion dynamics through microscopic processes in active Brownian particles. Sci. Rep. 10(1), 1-7 (2020)

24. S. Ghosh, S. Bhattacharya, Computational model on covid-19 pandemic using probabilistic cellular automata. SN Comput. Sci. 2(3), 1-10 (2021)

25. S. Ghosh, S. Bhattacharya, A data-driven understanding of covid-19 dynamics using sequential genetic algorithm based probabilistic cellular automata. Appl. Soft Comput. 96, 106692 (2020)

26. T. Caraballo, S. Keraani, Analysis of a stochastic sir model with fractional Brownian motion. Stoch. Anal. Appl. 36(5), 895-908 (2018)

27. R. Van der Hofstad, A.J.E.M. Janssen, J.S.H. Van Leeuwaarden, Critical epidemics, random graphs, and Brownian motion with a parabolic drift. Adv. Appl. Probab. 42(4), 1187-1206 (2010)
28. A. Miao, T. Zhang, J. Zhang, C. Wang, Dynamics of a stochastic sir model with both horizontal and vertical transmission. J. Appl. Anal. Comput 8(4), 1108-1121 (2018)

29. H. De-Leon, F. Pederiva, Particle modeling of the spreading of coronavirus disease (covid-19). Phys. Fluids 32(8), 087113 (2020)

30. N. Fabiano, S. Radenovic, Geometric Brownian motion and a new approach to the spread of covid-19 in Italy. Gulf J. Math. 10(2), 25-30 (2021)

31. Y. Zhao, C. Huepe, P. Romanczuk. Contagion dynamics in self-organized systems of self-propelled agents. arXiv preprint arXiv:2103.12618, (2021)

32. S.M. Moghadas, M.C. Fitzpatrick, P. Sah, A. Pandey, A. Shoukat, B.H. Singer, A.P. Galvani, The implications of silent transmission for the control of covid-19 outbreaks. Proc. Natl. Acad. Sci. 117(30), 17513-17515 (2020)

33. J. Hellewell, S. Abbott, A. Gimma, N.I. Bosse, C.I. Jarvis, T.W. Russell, J.D. Munday, A.J. Kucharski, W.J. Edmunds, F. Sun et al., Feasibility of controlling covid-19 outbreaks by isolation of cases and contacts. Lancet Glob. Health 8(4), e488-e496 (2020) 